\title{
Therapeutic Effects of Nrf2 Activation by Bardoxolone Methyl in Chronic Heart Failure ${ }^{\mathbb{s}}$
}

\author{
Changhai Tian, Lie Gao, Andi Zhang, Bryan T. Hackfort, and Irving H. Zucker \\ Department of Cellular and Integrative Physiology, University of Nebraska Medical Center, Omaha, Nebraska \\ Received July 29, 2019; accepted October 10, 2019
}

\begin{abstract}
Oxidative stress plays an important role in the pathogenesis of chronic heart failure (CHF) in many tissues. Increasing evidence suggests that systemic activation of nuclear factor (erythroidderived 2)-like 2 (Nrf2) signaling can protect against postinfarct cardiac remodeling by reducing oxidative stress. However, it remains to be elucidated if Nrf2 activation exerts therapeutic effects in the CHF state. Here, we investigated the beneficial hemodynamic effects of bardoxolone methyl (2-Cyano-3,12dioxooleana-1,9(11)-dien-28-oic acid methyl ester, CDDO-Me), a pharmacological activator of Nrf2, in a rodent model of CHF. Based on echocardiographic analysis, rats at 12 weeks post-myocardial infarction (MI) were randomly split into four groups. CDDO-Me $(5 \mathrm{mg} / \mathrm{kg}$, i.p.) was administered daily for another 2 weeks in sham and CHF rats and compared with vehicle treatment. Echocardiographic and hemodynamic analysis suggest that short-term CDDO-Me administration increased stroke volume and cardiac output in CHF rats and decreased left ventricle end-diastolic pressure. Molecular studies revealed that CDDO-Me-induced cardiac functional improvement was
\end{abstract}

\section{Introduction}

The imbalance between pro- and antioxidant pathways is known to be important in the progression of chronic heart failure (CHF) in the post-myocardial infarction (MI) state (Bhimaraj and Tang, 2012; Farias et al., 2017). Nuclear factor (erythroidderived 2)-like 2 ( Nrf2) as a master transcription factor is expressed in most tissues to act as an amplifier of antioxidant pathways and has been associated with the expression of antioxidant enzymes in the myocardium (Tan et al., 2011; Ma, 2013; Tian et al., 2018). Our previous work has shown that myocardial Nrf2 protein is decreased in the CHF state (Tian et al., 2018), thereby potentially disrupting redox balance and contributing to myocardial as well as remote organ dysfunction.

This work was supported by the National Institutes of Health National Heart, Lung, and Blood Institute [Grant P01HL62222 (I.H.Z.)], the American Heart Association Career Development Award [19CDA34520004 (C.T.)], and the Undergraduate Summer Research Fellowship program through the American Physiological Society (A.Z.).

The authors have no conflict of interest.

https://doi.org/10.1124/jpet.119.261792.

S This article has supplemental material available at jpet.aspetjournals.org. attributed to an increase of both Nrf2 transcription and translation, and a decrease of oxidative stress in the noninfarcted areas of the heart. Furthermore, CDDO-Me reduced NF- $\kappa \mathrm{B}$ binding and increased Nrf2 binding to the CREB-binding protein, which may contribute to the selective increase of Nrf2 downstream targets, including NADPH Oxidase Quinone 1, Heme Oxygenase 1, Catalase, and Glutamate-Cysteine Ligase Catalytic Subunit, and the attenuation of myocardial inflammation in $\mathrm{CHF}$ rats. Our findings suggest that Nrf2 activation may provide beneficial cardiac effects in MI-mediated CHF.

\section{SIGNIFICANCE STATEMENT}

Chronic heart failure (CHF) is the leading cause of death among the aged worldwide. The imbalance between pro- and antioxidant pathways is a determinant in the pathogenesis of CHF. Systemic activation of Nrf2 and antioxidant protein signaling by bardoxolone methyl may have beneficial effects on cardiac function and result in improvements by enhancing antioxidant enzyme expression and attenuating myocardial inflammation.

Nrf2 protein is repressed by Kelch-like ECH-associated protein 1, which is a redox-sensitive substrate adaptor for the Cullin-3 E3 ubiquitin ligase. Modification of cysteine residues in Kelch-like ECH-associated protein 1 by electrophilic compounds prevents ubiquitylation of Nrf2, allowing de novo synthesized Nrf2 to accumulate in the nucleus, where it transactivates genes that contain antioxidant response element (ARE) sequences and competes with NF-kB for binding to CREB-binding protein (CBP) (Kwak et al., 2002; Li et al., 2008; Liu et al., 2008; Kim et al., 2013; Tebay et al., 2015). Increasing evidence has demonstrated the potential beneficial effects of Nrf2 activation in a variety of animal models of cardiovascular disease (Appendino et al., 2011; Bai et al., 2015, 2017; Qian et al., 2015; Xin et al., 2018; Wafi et al., 2019).

Using a cardiac-specific mouse Nrf2 transgenic model, cardiac-specific Nrf2 expression was observed to be important in maintaining redox homeostasis and preventing oxidative stress and subsequent pathologic remodeling in a model of isoproterenol-induced heart failure (Shanmugam et al., 2019). Global deletion of Nrf2 in mice promoted the pathogenesis of heart failure by increasing oxidative damage in the heart

ABBREVIATIONS: ARE, antioxidant response element; CBP, CREB-binding protein; CDDO-Me, bardoxolone methyl (2-Cyano-3,12-dioxooleana1,9(11)-dien-28-oic acid methyl ester ); CHF, chronic heart failure; DH404, dihydro-CDDO-trifluoroethyl amide; eNOS, endothelial nitric oxide synthase; Grx1, Glutaredoxin-1; HO-1, Heme Oxygenase 1; LVEDP, left ventricle end-diastolic pressure; MI, myocardial infarction; NIH, National Institutes of Health; NQO1, NADPH Oxidase Quinone 1; Nrf2, nuclear factor (erythroid-derived 2)-like 2; Veh, vehicle. 
(Li et al., 2009). Our laboratory has shown that when Nrf2 was selectively deleted in the rostral ventrolateral medulla of the brain stem, antioxidant enzyme expression was reduced and oxidative stress was significantly increased, thus potentially contributing to sympatho-excitation in CHF (Gao et al., 2017). On the other hand, in the post-myocardial infarction heart failure state, selectively upregulating Nrf2 in this region reduced sympathetic outflow (Ma et al., 2019).

Currently, several Nrf2 activators have been examined in disease states and are now being actively promoted in clinical trials. Many of these studies focus on renal disease, skeletal muscle abnormalities, and multiple sclerosis (Cuadrado et al., 2019). There have been few studies to investigate the effects of Nrf2 activators on cardiac functions in a model of $\mathrm{CHF}$. In the current study, our objective was to determine if the smallmolecule Nrf2 activator bardoxolone methyl (CDDO-Me), the methyl ester of the synthetic triterpenoid 2-cyano-3,12-dioxooleana-1,9(11)-dien-28-oic acid altered hemodynamics and antioxidant enzyme expression in the myocardium of rats with post-MI CHF. CDDO-Me, a prototype of a class of triterpenoid compounds, has been used to treat chronic kidney disease in a phase 3 clinical trial (Pergola et al., 2011) and pulmonary hypertension in a phase 2 clinical trial (Hu et al., 2015). Unfortunately, because CDDO-Me did not prevent the progression of renal disease and increased mortality from cardiovascular events in patients with type 2 diabetes mellitus and stage 4 chronic kidney disease, these clinical trials were terminated (de Zeeuw et al., 2013; Chertow and de Zeeuw, 2014). However, CDDO-Me still demonstrated a potential to reduce oxidative stress, decrease inflammatory mediators (Ruiz et al., 2013; Zoja et al., 2014; Pickering et al., 2018), and prevent the cardiac and renal pathophysiology induced by high-fat diet in mice (Camer et al., 2016).

Recently, a CDDO-Me derivative, dihydro-CDDO-trifluoroethyl amide (DH404), has been shown to provide significant attenuation of pathologic cardiac remodeling in CHF rats by decreasing the glutathionylation of endothelial nitric oxide synthase (eNOS) and increasing the physical interaction between eNOS and Glutaredoxin-1 (Grx1) (Bubb et al., 2017), suggesting that CDDO-Me may have clinical benefits for protecting the myocardium from pathophysiological remodeling. Therefore, we hypothesized that chronic lowdose treatment with CDDO-Me would result in improved ventricular function by activating Nrf2/ARE signaling and increasing antioxidant enzyme levels in the myocardium of MI-induced CHF rats.

\section{Materials and Methods}

All animal studies were carried out with the approval of the University of Nebraska Medical Center Institutional Animal Care and Use Committee and followed the National Institutes of Health (NIH) Guide for the Care and Use of Laboratory Animals.

Animals and Heart Failure Model. Animals were group housed in the Department of Comparative Medicine at the University of Nebraska Medical Center, and were subjected to a 12-hour light/dark cycle at approximately $25^{\circ} \mathrm{C}$. MI was created by ligating the left coronary artery following routine procedures in our laboratory and as described previously (Gao et al., 2005; Wang et al., 2010). In brief, male Sprague-Dawley rats (body weight: $180-200 \mathrm{~g}$ ) were anesthetized with $2 \%$ isoflurane in oxygen, intubated, and placed on positivepressure ventilation. After opening the chest and removing the pericardium, the left anterior descending coronary artery was ligated with 5-0 silk suture in $\mathrm{CHF}$ animals, whereas the same procedures were followed except the coronary artery was not ligated in sham animals. Following surgery, animals recovered in a heated cage until recumbent and moving. After 24 hours, they were generally eating and drinking normally and were treated with $5 \mathrm{mg} / \mathrm{kg}$ carprofen and $0.1 \mathrm{mg} / \mathrm{kg}$ buprenorphine for 3 postoperative days. Twelve weeks post MI, cardiac function was evaluated by echocardiography. Rats were considered to be in CHF when the ejection fraction was less than $40 \%$.

Sham and CHF rats were given vehicle (DMSO; $0.1 \mathrm{ml}$, i.p.) or CDDO-Me (5 mg/kg, $0.1 \mathrm{ml}$ i.p.; APExBio, Houston, TX), respectively, daily starting at 12 weeks post MI or post sham surgery as outlined in Fig. 1 based on the first echo analysis.

Infarct Size. To evaluate the infarct size, the CHF rats were euthanized with pentobarbital $(150 \mathrm{mg} / \mathrm{kg}$, i.p.). The left ventricle showing the transmural infarct was photographed, and the borders of the infarct and entire left ventricle were outlined. The scar borders were clearly visible in all infarcted animals. The areas were measured using ImageJ (NIH) software. The infarct size was calculated as the percentage of the entire left ventricle.

Echocardiography. Assessment of cardiac function was carried out using high-frequency echocardiography (Vevo 3100, 15-MHz probe; Visual Sonics, Inc., Toronto, Canada). Under light isoflurane anesthesia, rats were placed on a heated platform that recorded EKG, body temperature, and respiratory rate. The thorax was shaved and treated with a depilatory to remove hair. Images were acquired in the two-dimensional mode using the long axis view. An M-mode image was acquired using the two-dimensional image to define a section at the level of the papillary muscles. In addition, an EKG-gated Kilohertz Visualization image was acquired in the long axis to obtain high-fidelity averaged beat data. All cardiac parameters were analyzed using Visual Sonics software.

$1^{\text {st }}$ Echo analysis and group animals

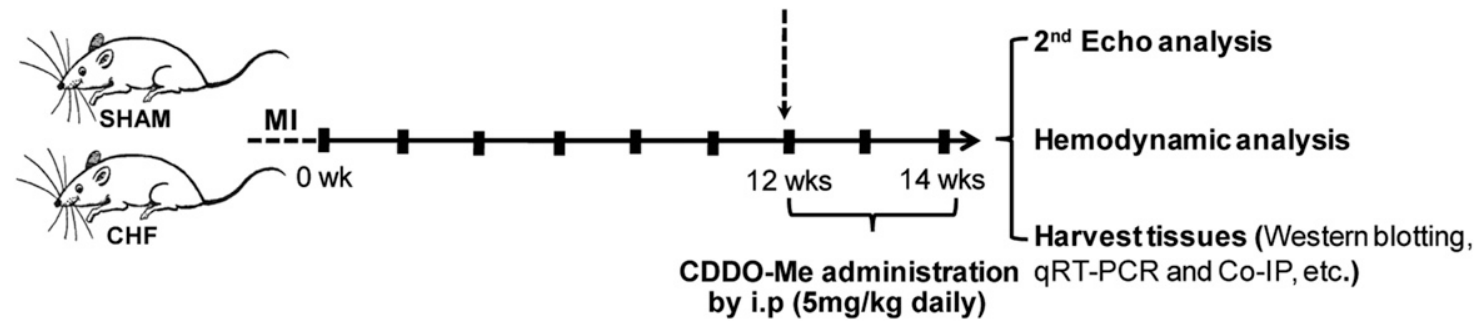

Fig. 1. Outline of the experimental protocol. Rats were allowed to recover for 12 weeks after thoracic surgery. At this time, an echocardiogram was taken, and treatment with either vehicle or CDDO-Me was begun for 2 weeks. At 14 weeks post thoracic surgery, a second echocardiogram was taken, and hemodynamic measurements were determined and tissues taken for biochemical analyses. Co-IP, coimmunoprecipitation; qRT-PCR, quantitative reverse-transcription polymerase chain reaction; i.p, intraperitoneal; Echo, Echocardiogram. 
TABLE 1

Echocardiographic analysis of CHF rats treated with CDDO-Me at 14 weeks post thoracic surgery

\begin{tabular}{lcccc}
\hline Parameters & Sham $+\operatorname{Veh}(n=7)$ & Sham+CDDO-Me $(n=7)$ & CHF+Veh $(n=8)$ & CHF+CDDO-Me $(n=8)$ \\
\hline Volume; s $(\mu \mathrm{l})$ & $189.4 \pm 83.9$ & $213.6 \pm 77.0$ & $537.0 \pm 136.9^{*}$ & $570.0 \pm 279.5$ \\
Volume; $(\mu \mathrm{l})$ & $596.3 \pm 231.3$ & $546.4 \pm 188.3$ & $820.0 \pm 226.0$ & $953.1 \pm 391.5$ \\
Stroke volume $(\mu \mathrm{l})$ & $406.9 \pm 155.5$ & $332.8 \pm 116.1$ & $232.1 \pm 52.4^{* * *}$ & $425.2 \pm 249 * * *$ \\
Ejection fraction (\%) & $68.5 \pm 4.1$ & $60.7 \pm 3.8$ & $34.2 \pm 8.5^{\xi}$ & $39.2 \pm 15.9$ \\
Fractional shortening (\%) & $29.7 \pm 11.4$ & $31.8 \pm 12.6$ & $13.8 \pm 7.2^{\psi}$ & $18.2 \pm 7.0$ \\
Cardiac output (ml/min) & $142.7 \pm 58.3$ & $152.5 \pm 15.7$ & $30.7 \pm 85.7^{\omega}$ & $174.3 \pm 81.6^{\triangle}$ \\
Infarct size (\%) & - & - & $36.2 \pm 8.3$ & $29.6 \pm 8.1$ \\
\hline
\end{tabular}

${ }^{*} P<0.0001$ vs. Sham + Veh; ${ }^{* *} P<0.03$ vs. Sham + Veh; $* * * * P 0.05$ vs. CHF + Veh; ${ }^{\xi} P<0.0001$ vs. Sham + Veh; ${ }^{\psi} P<0.01$ vs. Sham + Veh; ${ }^{\omega} P<0.02$ vs. Sham + Veh; ${ }^{\triangle} P<0.01$ vs. CHF+CDDO-Me.

Hemodynamics. At the end of the study, experimental rats were anesthetized with isoflurane. A $2 \mathrm{~F}$ micromanometer-tipped pressure transducer catheter (SPR-407; Millar Instruments, Houston, TX) was inserted into the right carotid artery to measure arterial blood pressure and left ventricular pressure. Pressure signals were recorded on a Powerlab S16 using Laboratory Chart 7 software (ADInstruments, Inc., Colorado Springs, CO), from which heart rate, left ventricular end-diastolic pressure (LVEDP), left ventricular peaksystolic pressure, and the maximum rate of increase and decrease of left ventricular pressure (dp/dt $t_{\max }$ and $\left.\mathrm{dp} / \mathrm{dt}_{\min }\right)$ were derived.

RNA Extraction and Quantitative Reverse-Transcription Polymerase Chain Reaction (qRT-PCR). Myocardial tissues were subjected to RNA extraction using RNeasy Mini Kit (QIAGEN Inc., Valencia, CA) per the manufacturer's recommendations. cDNA synthesis was carried out using reverse-transcription MasterMix (Applied Biologic Materials, Richmond, Canada). SYBR Select Master Mix (Life Technologies, Los Angeles, CA) and the specified primer pairs (Integrated DNA Technologies; see Table 1) were used to amplify samples with the StepOnePlus Real Time System (Applied Biosystems, Waltham, MA). The quantification of mRNA expression was performed with the $2^{(-\Delta \Delta \mathrm{Ct})}$ method.

Western Blotting Analysis. Myocardial tissue was collected from noninfarcted regions in CHF rats and the corresponding left ventricular regions in the Sham group. All samples were directly subjected to protein extraction by homogenization in Radioimmunoprecipitation assay (RIPA) buffer supplemented with Halt Protease Inhibitor Cocktail (100×; Thermo Fisher Scientific, Rockford, IL). The protein concentration of each fraction was determined by BCA Protein Assay (Thermo Fisher Scientific). The protein samples were separated by SDS-PAGE gel and then transferred onto a Polyvinylidene fluoride
(PVDF) membrane, blocked with 5\% nonfat dry milk in PBS, and then incubated overnight at $4^{\circ} \mathrm{C}$ with the following primary antibodies: Nrf2 (1:1000; ab137550; Abcam), GAPDH (glyceraldehyde-3-phosphate dehydrogenase) (1:10,000; MA5-15738; Thermo Fisher Scientific), Heme Oxygenase 1 (1:1000; ab68477), NADPH Oxidase Quinone 1 (NQO1; 1:1000; ab80588), Catalase (1:1000; AF3398; R\&D Systems), and SOD2 (Superoxide Dismutase 2) (FL-222) (1:1000; sc-30080). After washing with PBST, the blots were then incubated with secondary antibodies (1:5000; Thermo Scientific, Waltham, MA). The blots were washed with PBST (1X Phosphate-Buffered Saline, $0.1 \%$ Triton $\mathrm{X}-100$ ) and then subjected to chemiluminescent substrate and the UVP Bioimaging Systems (UVP LLC, Upland, CA) for imaging and quantification.

Coimmunoprecipitation. Noninfarcted regions of left ventricles in CHF rats (14 weeks post MI) and the corresponding left ventricular regions in the sham group were collected and subjected to homogenization with Radioimmunoprecipitation assay (RIPA) buffer supplemented with Halt Protease Inhibitor Cocktail (Thermo Fisher Scientific) and serial centrifugation to clear the protein samples. After the normalization of protein concentration by BCA Protein Assay, the protein samples were incubated with CBP antibody (sc-7300; Santa Cruz Biotechnology, Dallas, TX) overnight at $4^{\circ} \mathrm{C}$. The immune complexes were incubated with Protein A/G PLUSAgarose (sc-2003; Santa Cruz Biotechnology) at room temperature for 2 hours, and then subjected to western blotting analysis with the following primary antibodies: Nrf2 (ab31163; Abcam) and NF- $\kappa$ B (ab7970), respectively. An aliquot of the lysates was used as an input and loading control.

Histologic and Immunochemical Analysis. Hearts were perfused with ice-cold PBS and frozen on dry ice. Frozen sections of the

TABLE 2

Primer pairs used for quantitative reverse-transcription polymerase chain reaction analysis (qRT-PCR)

\begin{tabular}{|c|c|c|}
\hline Gene & Sequence & Gene Bank Number \\
\hline \multirow[t]{2}{*}{ Nrf2 } & Forward: 5'-GCCAGCTGAACTCCTTAGAC-3' & NM_031789.2 \\
\hline & Reverse: 5'-GATTCGTGCACAGCAGCA - -3' & \\
\hline \multirow[t]{2}{*}{$\mathrm{HO}-1$} & Forward: 5'-CGACAGCATGTCCCAGGATT-3' & NM 012580.2 \\
\hline & Reverse: 5-TCGCTCTATCTCCTCTTCCAGG-3' & \\
\hline \multirow[t]{2}{*}{$N Q O 1$} & Forward: 5'-CATTCTGAAAGGCTGGTTTGA-3' & NM_017000.3 \\
\hline & Reverse: 5'-CTAGCTTTGATCTGGTTGTCG-3' & \\
\hline \multirow[t]{2}{*}{ SOD1 } & Forward: 5'-GCGGTGAACCAGTTGTGGTG-3' & NM_017050.1 \\
\hline & Reverse: 5'-GCTGGACCGCCATGTTTCTT-3' & \\
\hline \multirow{2}{*}{ SOD2 } & Forward: 5'-AGGAGAGTTGCTGGAGGCTA-3' & NM 017051.2 \\
\hline & Reverse: 5'-AGCGGAATAAGGCCTGTTGTT-3' & \\
\hline \multirow[t]{2}{*}{$C A T$} & Forward: 5'-CGACCGAGGGATTCCAGATG-3' & NM_012520.2 \\
\hline & Reverse: 5'-CCTGCCTCTTCAACAGGCAA-3' & \\
\hline \multirow[t]{2}{*}{$G C L C$} & Forward: 5'-GTGGACACCCGATGCAGTAT-3' & NM_012815.2 \\
\hline & Reverse: 5'-TCATCCACCTGGCAACAGTC-3' & \\
\hline \multirow[t]{2}{*}{ Grx1 } & Forward: 5'-CTGTCAGCATGGCTCAGGAGT-3' & NM_022278.1 \\
\hline & Reverse: 5'-CCACAAATTCCAGGAGACCAC-3' & \\
\hline \multirow[t]{2}{*}{$G P X 1$} & Forward: 5'-CACAGTCCACCGTGTATGCC-3' & NM_030826.4 \\
\hline & Reverse: 5'-AAGTTGGGCTCGAACCCACC-3' & \\
\hline \multirow[t]{2}{*}{$T N F-\alpha$} & Forward: 5'-AAATGGGCTCCCTCTCATCAGTTC-3' & X66539.1 \\
\hline & Reverse: 5'-TCTGCTTGGTGGTTTGCTACGAC-3' & \\
\hline
\end{tabular}




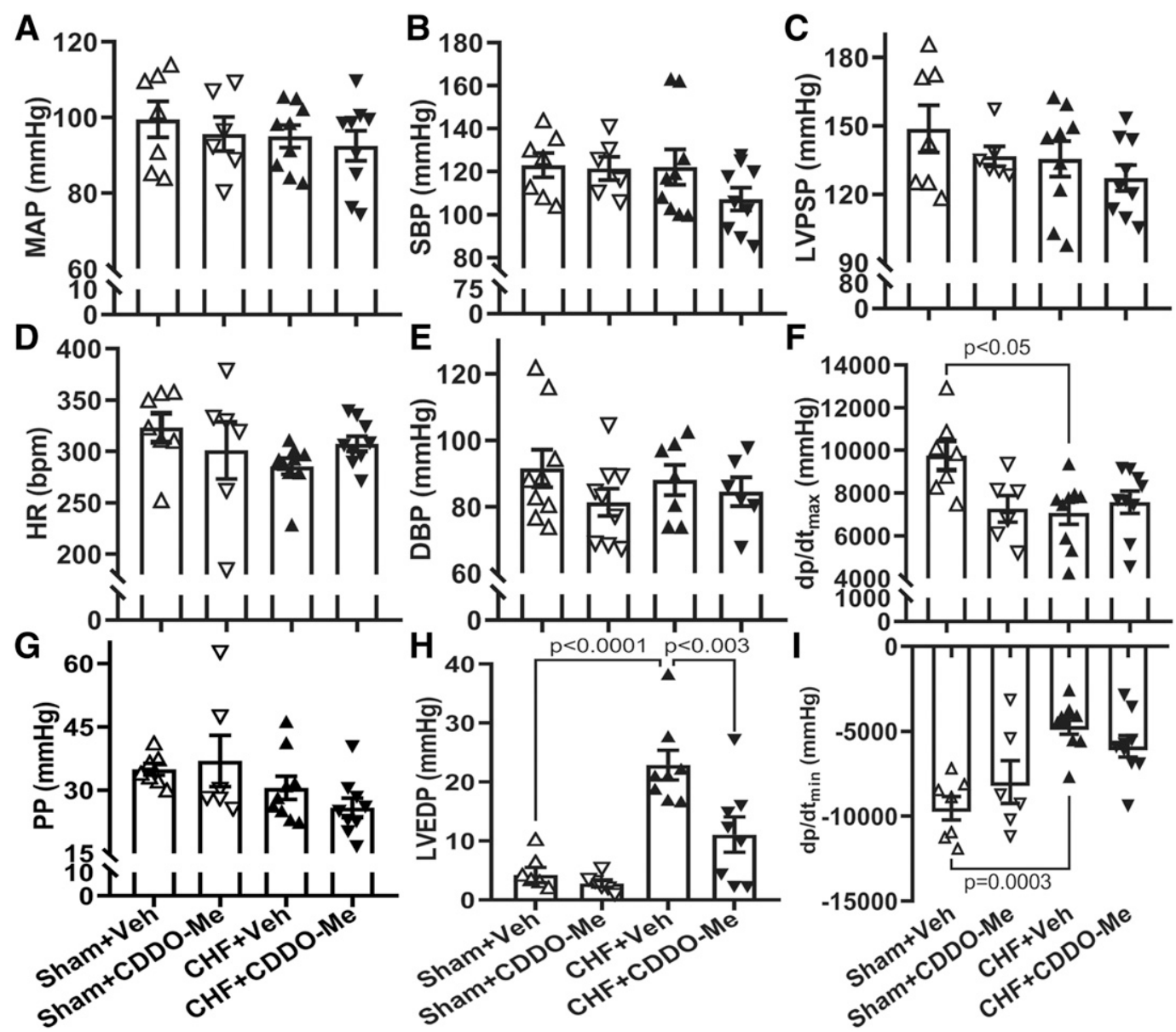

Fig. 2. Hemodynamic data from CHF rats treated with CDDO-Me. Blood pressure (A); systolic pressure (SBP) (B); left ventricular peak-systolic pressure (LVPSP) (C); heart rate (HR) (D); diastolic blood pressure (DBP) (E), and pulse pressure (PP) (G). No significant difference was observed between different groups. Left-ventricular maximum rate of pressure increase $\left(\mathrm{dp} / \mathrm{dt}_{\mathrm{max}}\right)$ and decrease $\left(\mathrm{dp} / \mathrm{dt}_{\mathrm{min}}\right)$ were significantly decreased in CHF rats treated with vehicle compared with that in sham rats treated with vehicle ( $\mathrm{F}$ and I), and LVEDP was significantly increased in Veh-treated CHF rats and partially restored after CDDO-Me treatment (H). Sham-Veh $(n=7)$, sham-CDDO-Me $(n=6)$, CHF-Veh $(n=8)$, CHF-CDDO-Me $(n=8)( \pm$ S.E.M.). MAP, mean arterial pressure.

left ventricle were prepared ( $10 \mu \mathrm{m}$, Leica CM 3050S Research Cryostat) and stored at $-20^{\circ} \mathrm{C}$ until staining. After fixation with $4 \%$ Paraformaldehyde (PFA) solution at room temperature for 30 minutes, the sections were permeabilized with $0.2 \%$ Triton $\mathrm{X}-100$ for 20 minutes and then blocked with $5 \%$ goat serum for 2 hours. After washing with PBS, the sections were incubated with the following primary antibodies: 4-HNE antibody (ab48506; Abcam, Cambridge, MA) and 8-OHdG antibody (sc393871; Santa Cruz Biotechnology, Inc., Santa Cruz, CA). The sections were then washed with PBS and subjected to fluorescence-conjugated secondary antibodies and nuclear staining with DAPI (4',6-diamidino-2phenylindole). Fluorescent images were taken by a Zeiss 710 confocal microscope (Carl Zeiss, Oberkochen, Germany). Quantification was done using ImageJ (NIH) software.

Statistics. All data are reported as the mean \pm S.E.M. Statistical significance was considered when $P$ values $<0.05$ were determined by Student's $t$ test for two different groups or one-way analysis of variance followed by Tukey's post hoc test for multiple group comparisons, where appropriate, using GraphPad Prism 8.0 (GraphPad Software, San Diego, CA).

\section{Results}

CDDO-Me Promotes Nrf2 Nuclear Translocation and Improves Heart Hemodynamics in CHF Rats. Nrf2 activation was assessed by Nrf2 nuclear translocation after CDDO-Me treatment. Subcellular fractionation was performed with cardiac and skeletal muscle. Western blot results demonstrated that CDDO-Me systemically induced Nrf2 nuclear translocation in the heart and skeletal muscle (Supplemental Fig. 1) and induced the increase of Nrf2 and its target transcripts in a dose-dependent manner (Supplemental Fig. 2).

Echocardiographic data are shown in Table 2. After 2 weeks of treatment, the infarct size in the CDDO-Me-treated CHF group showed a trend to decrease compared with vehicletreated (from $36.2 \pm 8.3$ to $29.6 \pm 8.1$ ), although this was not significant. The left ventricular systolic volume in the $\mathrm{CHF}+$ vehicle (Veh) group was significantly higher than that in the sham +Veh group; however, there was no significant difference in left ventricular volumes between the sham and CHF groups after CDDO-Me treatment. CDDO-Me treatment had no significant effects on echocardiographic parameters in the sham group. On the other hand, CDDO-Me significantly increased stroke volume and cardiac output in the CHF group. In addition, hemodynamic analyses done at 14 weeks post MI demonstrated that there were significant decreases in $\mathrm{dp} /$ $\mathrm{dt}_{\max / \min }$ (Fig. 2, F and I) and a significant increase in LVEDP in the $\mathrm{CHF}+$ Veh group (Fig. $2 \mathrm{H}$ ), typical of the heart failure state. Although CDDO-Me had no effects on maximal and minimal dp/dt in sham and CHF animals (Fig. 2, F and I), the CDDO-Me-treated CHF group displayed a significantly lower LVEDP as compared with the vehicle-treated CHF group 

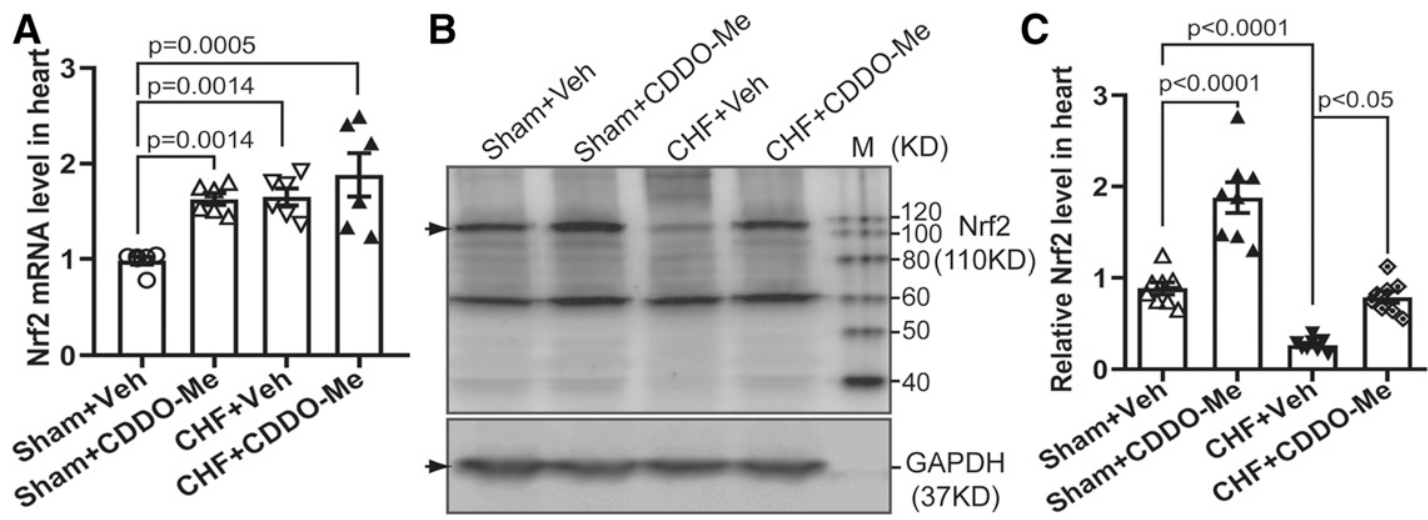

Fig. 3. CDDO-Me elevated both mRNA and protein levels of Nrf2 in the infarcted heart. (A) Quantitative reverse-transcription polymerase chain reaction data show CDDO-Me increases the Nrf2 transcription in the heart ( $n=6$, \pm S.E.M.). Typical immunoblots $(\mathrm{B})$ and pooled data (C) showing CDDO-Me induced the translational increase of Nrf2 in the noninfarcted left ventricle 2 weeks post CDDO-Me administration $(n=7, \pm$ S.E.M.). GAPDH, glyceraldehyde-3-phosphate dehydrogenase.

(Fig. 2H), suggesting some benefit of CDDO-Me to the failing heart. Moreover, CDDO-Me had no significant effects on mean arterial pressure (Fig. 2A), heart rate (Fig. 2D), systolic pressure (Fig. 2B), diastolic blood pressure (Fig. 2E), pulse pressure (Fig. 2G), or left ventricular peak-systolic pressure (Fig. 2C).

CDDO-Me Induced the Transcriptional and Translational Activation of Nrf2 in CHF. To confirm the activation of Nrf2/ARE signaling by CDDO-Me in CHF rats, we determined myocardial Nrf2 mRNA levels in four different groups. CDDO-Me treatment increased Nrf2 mRNA levels in both sham and CHF groups (Fig. 3A). However, there were no differences between CHF rats treated with vehicle versus CDDO-Me. Although there was also a significant increase in Nrf2 mRNA in the CHF group with vehicle treatment, Nrf2 protein was significantly decreased in vehicle-treated CHF rats, which was partially reversed by CDDO-Me treatment
(Fig. 3, B and C). To further confirm the activation of Nrf2/ ARE signaling after CDDO-Me treatment, we evaluated the transcriptional and translational levels of some downstream targets of Nrf2. The results demonstrated that there were significant increases of several antioxidant enzyme mRNA levels in the CDDO-Me-treated CHF group compared with the vehicle-treated CHF group, including Heme Oxygenase 1 (HO-1), NQO1, catalase, and glutamate-cysteine ligase. The effects of CDDO-Me on the transcription of antioxidant enzymes in the CHF group are markedly greater than that in the sham group (Fig. 4, A-D). However, other potential targets of Nrf2, including SOD1, SOD2, Grx1, and Gpx1, showed no significant differences (Fig. 4, E and F). Consistently, western blot analysis of some antioxidant enzymes also demonstrated that CDDO-Me selectively stimulated an increase of some antioxidant proteins, including $\mathrm{HO}-1, \mathrm{NQO} 1$, and catalase but not SOD2 (Fig. 5, A and B) in animals with CHF.
A

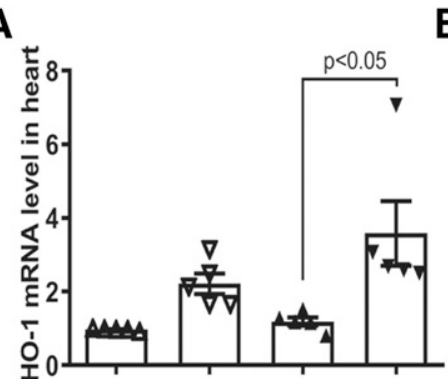

E

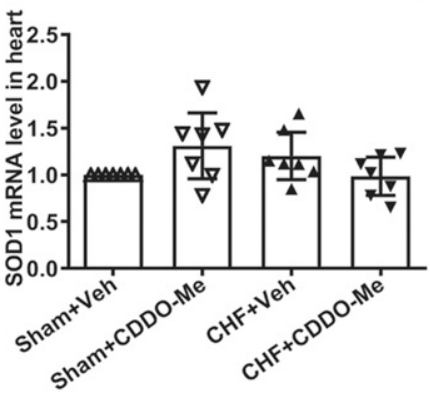

B

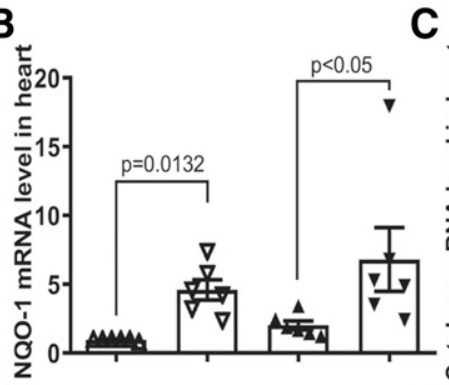

$\mathbf{F}$

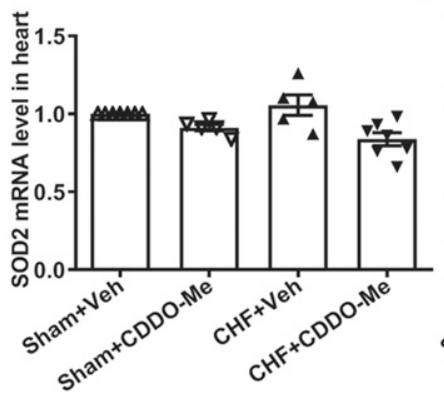

G
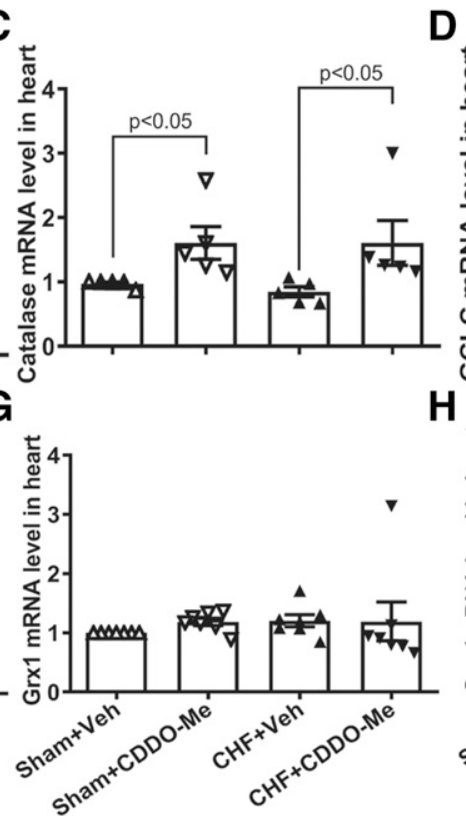

Fig. 4. Cardiac tissues from left ventricles were collected and then subjected to quantitative reverse-transcription polymerase chain reaction analysis with specific primers for HO-1 (A), NQO1 (B), catalase (C), glutamate-cysteine ligase (GCLC) (D), SOD1 (E), SOD2 (F), Grx1 (G), and Gpx1 (H). Glyceraldehyde-3-phosphate dehydrogenase (GAPDH) was used as an internal control ( $n=5$ to 6 , \pm S.E.M.). 
A

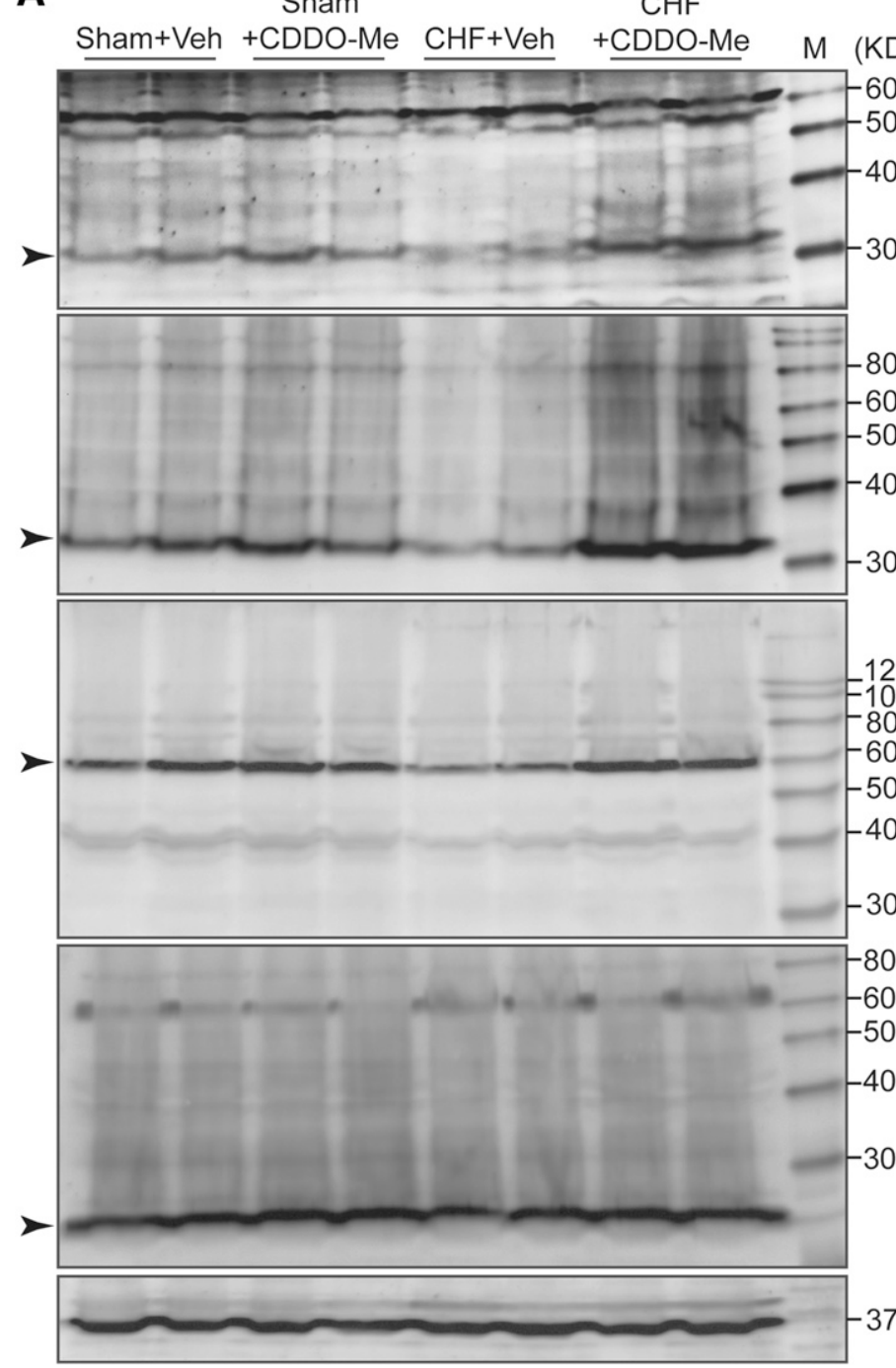

B
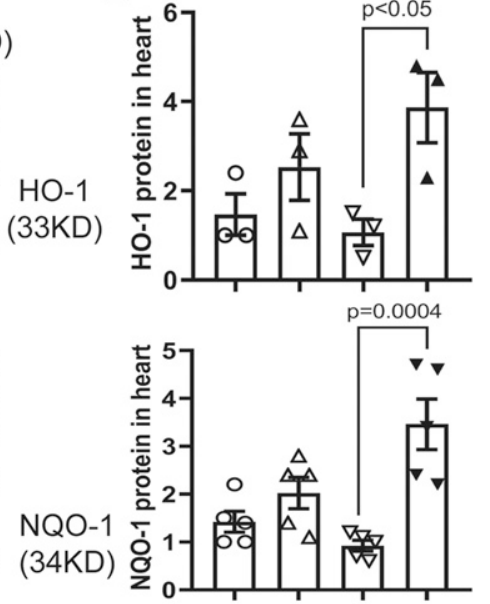

NQO-

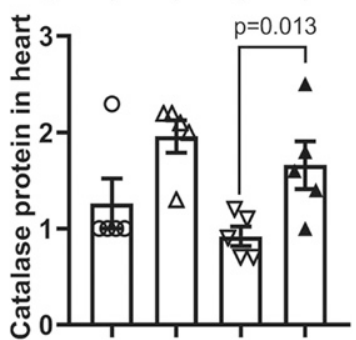

SOD2 $(25 K D)$

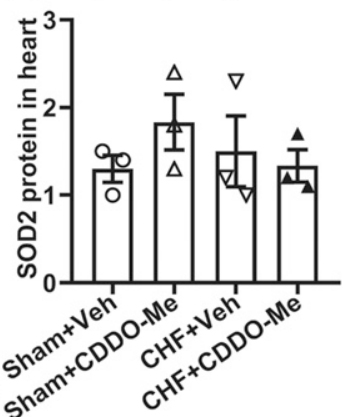

Fig. 5. Sham and CHF rats were treated with vehicle or CDDO-Me, respectively, for 2 weeks. The noninfarcted area of the left ventricles were collected and subjected to western blotting analysis for HO-1, NQO1, catalase, and SOD2. Glyceraldehyde-3-phosphate dehydrogenase (GAPDH) was used as a loading control (A); mean data are shown in (B) (士S.E.M.) as the ratio of each protein to GAPDH.

CDDO-Me Enhances Nrf2 Binding to CBP and Reduces the Binding of NF- $\kappa$ B to CBP. In addition to activating antioxidant enzyme expression by binding to the AREs on antioxidant promoters, Nrf2 has been shown to reduce inflammatory mediators by competing with $\mathrm{NF}-\mathrm{kB}(\mathrm{Li}$ et al., 2008; Liu et al., 2008; Kim et al., 2013). To determine if CDDO-Me reduced the NF- $\kappa$ B binding to the CBP, we carried out coimmunoprecipitation experiments with the CBP antibody using myocardial tissues, and then blotted with either $\mathrm{NF}-\kappa \mathrm{B}$ or Nrf2 antibodies, respectively. As shown in Fig. 6, the binding of NF- $\kappa \mathrm{B}$ to the $\mathrm{CBP}$ was significantly increased in $\mathrm{CHF}$ rats, whereas it was markedly reduced by CDDO-Me treatment. In contrast, CDDO-Me promoted a marked increase of Nrf2 binding to $\mathrm{CBP}$ in the $\mathrm{CHF}$ group, corresponding to the reduction of $\mathrm{NF}-\kappa \mathrm{B}$ binding to $\mathrm{CBP}$. However, CDDO-Me had no significant effects on NF- $\kappa$ B or Nrf2 binding to the $\mathrm{CBP}$ in sham rats (Fig. 6, A and B). Consistent with these findings, the enhanced binding of NF- $\kappa \mathrm{B}$ to $\mathrm{CBP}$ significantly promoted the production of the proinflammatory cytokine TNF- $\alpha$ in the myocardium of left ventricle in the vehicle-treated CHF group, which was attenuated by CDDOMe treatment (Fig. 6C). In addition, myocardial oxidative stress, including lipid peroxidation indicated by 4-HNE staining (Fig. 7, A and B) and DNA/RNA oxidation indicated by 8-OHdG staining (Fig. 7, C and D), was significantly increased in the vehicle-treated $\mathrm{CHF}$ group, which was attenuated by CDDO-Me treatment.

\section{Discussion}

In the current study, we clearly show that systemic administration of CDDO-Me improved cardiac functions, including stroke volume and cardiac output in CHF rats, and resulted in a decrease in LVEDP. The latter finding is of extreme importance since elevated LVEDP leads to pulmonary congestion and ultimately death in the CHF state. Moreover, CDDO-Me not only upregulated the expression of antioxidant enzymes to reduce myocardial oxidative stress in the myocardium of $\mathrm{CHF}$ rats by promoting the transcription and translation and subsequent nuclear translocation of Nrf2, 


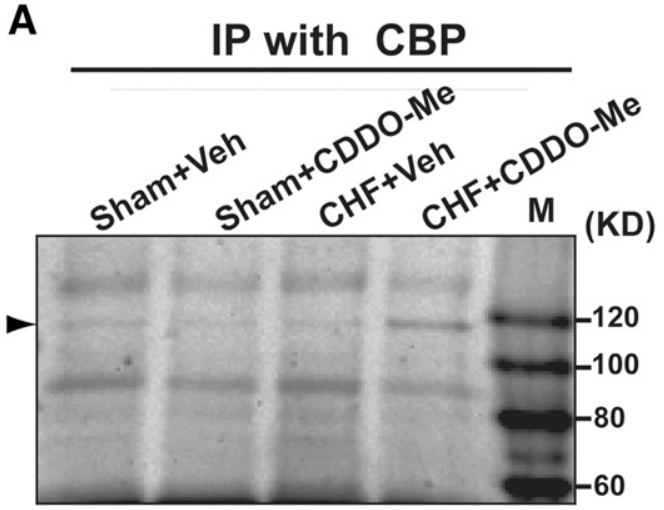

IB: Nrf2 (110 KD)

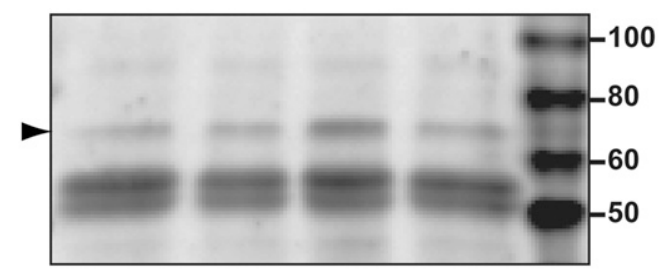

IB: NF- $\kappa B(65 \mathrm{KD})$

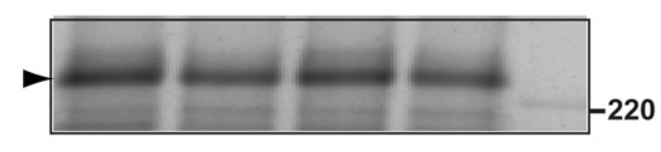

IB: CBP (265 KD)

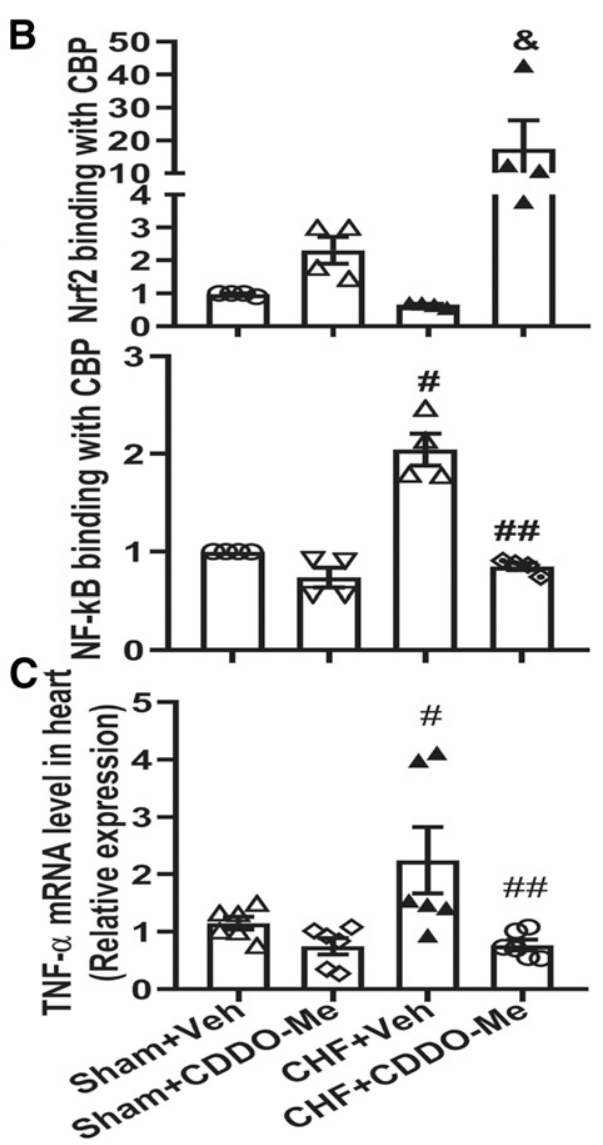

Fig. 6. Left ventricular tissue was subjected to coimmunoprecipitation with CBP antibody and then western blotting analysis with Nrf2 and NF-kB (p65) antibody, respectively. Immunoblots (A) and pooled data (B) showing the enhanced binding of Nrf2 protein to CBP and reduced binding of NF- $\mathrm{kB}$ to $\mathrm{CBP}$ in the noninfarcted left ventricle 2 weeks post CDDO-Me administration $(n=4$, \pm S.E.M.). ${ }^{\&} P<0.05$ vs. $\mathrm{CHF}+\mathrm{Veh} ;{ }^{\#} P<$ 0.0001 vs. sham +Veh; ${ }^{\# \#} P<0.0001$ vs. $\mathrm{CHF}+\mathrm{Veh}$. (C) The noninfarcted region of the left ventricle was collected and then subjected to RNA extraction and quantitative reverse-transcription polymerase chain reaction analysis with specific primers for TNF- $\alpha$. Quantitative reversetranscription polymerase chain reaction data show CDDO-Me inhibits the transcription of TNF- $\alpha$ in the infarcted heart compared with that in the Veh-treated CHF group $\left(n=6, \pm\right.$ S.E.M.). ${ }^{\#} P<0.05$ vs. sham + Veh; ${ }^{\# \#} P<0.05$ vs. CHF + Veh. IB, XXX; IP, immunoprecipitation; IB, immunoblotting. but also attenuated cardiac inflammation by preventing the physical binding of NF-kB p65 to CBP. Our findings strongly suggest that low-dose CDDO-Me has therapeutic potentials for improving cardiac function in the CHF state.

Cardiovascular dysfunction in the CHF state is multifactorial and involves almost every organ system. There is an increased oxidative stress in the progression of chronic heart failure, which is a major contributor during the development of CHF (Gutierrez et al., 2006; Santos et al., 2011). The dysregulation of antioxidant defense systems results in oxidative stress (Tsutsui et al., 2006; Canton et al., 2011), thus affecting cardiac structure and function (Siwik et al., 1999, 2001; Costa et al., 2016). Nrf2 as a highly conserved transcription factor regulates the expression of many target genes involved in cellular defense systems by binding to the AREs in their promoter regions (Zhang et al., 2013). These target genes are involved in the pathogenesis of cardiovascular diseases through oxidative stress-mediated cardiac pathologic remodeling (Lu et al., 2008; Ashrafian et al., 2012). Nrf2 activation has resulted in beneficial effects in a variety of cardiovascular diseases (Satta et al., 2017). Although significant improvement of renal function was observed in chronic kidney disease in patients with type 2 diabetes, this clinical trial was prematurely terminated due to adverse effects (de Zeeuw et al., 2013; Chertow and de Zeeuw, 2014). In spite of these adverse effects, we hypothesized that low-dose CDDO$\mathrm{Me}$ ( $5 \mathrm{mg} / \mathrm{kg}$ per day) would be beneficial in experimental CHF. CDDO-Me evoked Nrf2 nuclear translocation and downstream target activation (Figs. 4 and 5; Supplemental Figs. 1 and 2). Although the maximal and minimal left ventricular $\mathrm{dp} / \mathrm{dt}$ and several other hemodynamic parameters did not significantly change in the CHF animals treated with CDDO-Me, LVEDP, a critical index of chronic heart failure (Nagendran et al., 2014), was significantly reduced as compared with the vehicletreated CHF group (Fig. 2H), suggesting a beneficial effect of CDDO-Me on the left ventricle. Although the underlying mechanism is not clear, it appeared that CDDO-Me did not directly improve myocardial contractility or the rate of relaxation. It is possible that the reduced LVEDP is due to the CDDO-Me-induced peripheral vasodilation (Wang et al., 2014) and/or a reduction in preload. Importantly, CDDO-Me in rats with $\mathrm{CHF}$ also showed a significant increase in stroke volume and cardiac output. Recently, using a derivative of CDDO-Me (DH404), a similar study in a rat model of myocardial ischemia-reperfusion injury, where DH404 was administered at an early time point for 28 days, resulted in structural and functional benefits, in part, by decreasing glutathionylation of eNOS and increasing the physical interaction between eNOS and Grx1 (Bubb et al., 2017). Our data are supportive of a recent study where enhanced cardiacspecific Nrf2 expression protected myocardial structure and function from pathologic remodeling through an increased expression of antioxidant enzymes and an improved redox environment (Shanmugam et al., 2019) in an isoproterenolinduced heart failure model.

The current study suggests that systemic administration of CDDO-Me may improve cardiac function through upregulation of antioxidant enzymes by a CDDO-Me-mediated Nrf2 activation, thus contributing to the attenuation of oxidative stress in the left ventricle (Figs. 3-5 and 7). In addition, Nrf2 


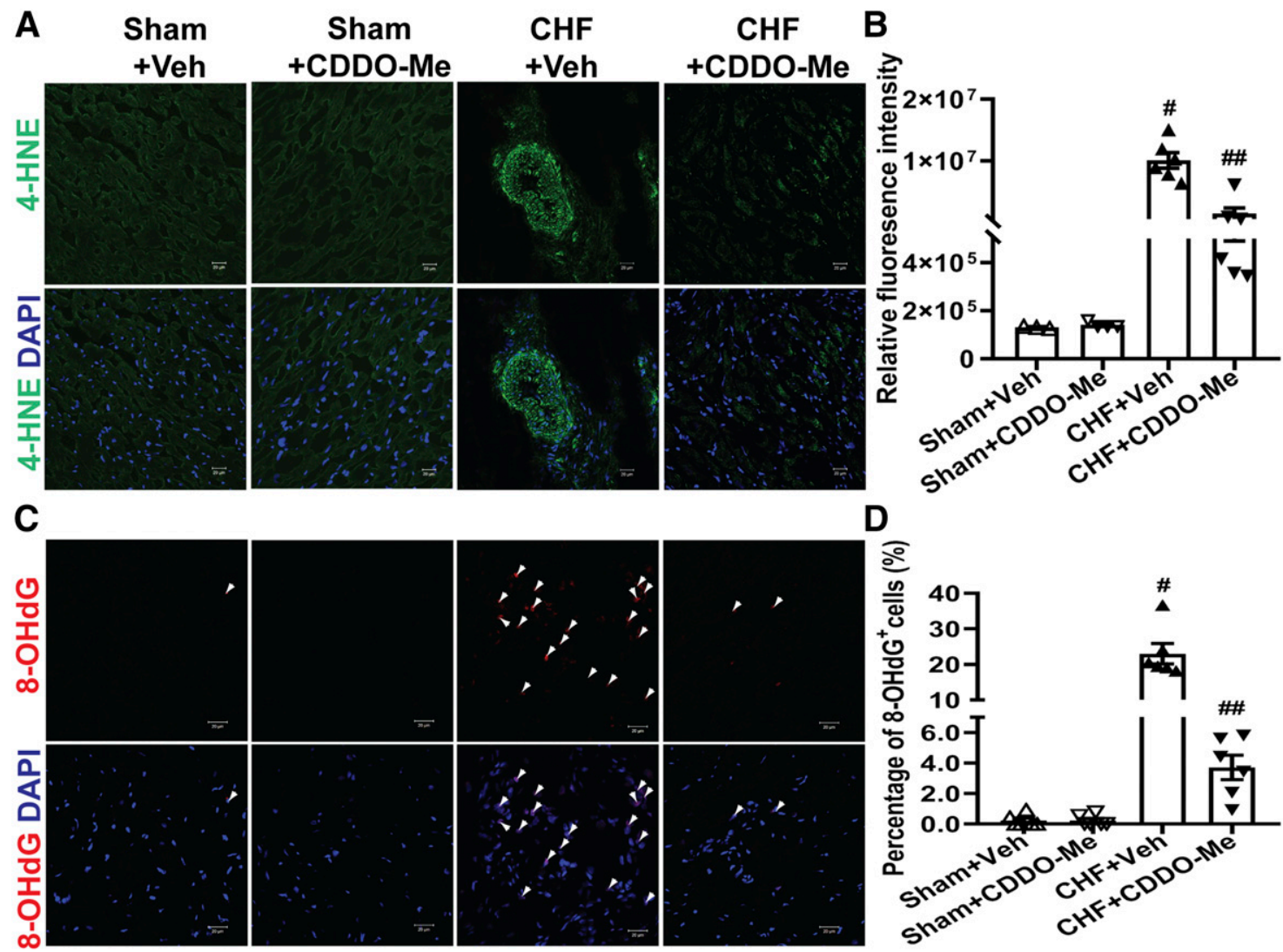

Fig. 7. CDDO-Me decreases oxidative stress of the heart. Oxidative stress in the left ventricles of hearts was determined in sham and CHF rats (12 weeks post MI) that were treated with vehicle or CDDO-Me for an additional 2 weeks, respectively. (A) Representative confocal microscopic images of the left ventricle with 4-HNE staining. 4-HNE-positive is shown in green. (B) The relative fluorescence intensities were quantified by ImageJ software $(\mathrm{NIH})($ sham +Veh: $n=3$; sham +CDDO-Me: $n=4 ; \mathrm{CHF}+$ Veh and CHF+CDDO-Me: $n=6) .{ }^{\#} P<0.0001$ vs. sham + Veh; ${ }^{\#} P<0.0001$ vs. CHF+Veh. $(\mathrm{C})$ Representative confocal microscopic images of the left ventricle 8-OHdG staining. 8-OHdG-positive is shown in red. (D) The percentage of 8-OHdG ${ }^{+}$cells was quantified (sham +Veh and sham +CDDO-Me: $n=4$; CHF+Veh and CHF+CDDO-Me: $n=6$ ). ${ }^{\#} P<0.0001 \mathrm{vs}$. sham + Veh; ${ }^{\# \#} P<0.0001 \mathrm{vs}$. $\mathrm{CHF}+$ Veh. Nuclei are shown in blue (DAPI, 4',6-diamidino-2-phenylindole). Original magnification, $400 \times$. All images were processed with the same confocal settings.

has also been shown to reduce inflammatory mediators by competing with NF-kB (Li et al., 2008; Liu et al., 2008; Kim et al., 2013). Consistently, we observed enhanced binding of Nrf2 to CBP in the heart in response to systemic administration of CDDO-Me in the CHF group, which prevented binding of NF- $\kappa$ B to CBP and subsequent inhibition of proinflammatory cytokine production (Fig. 6). In addition, it has been reported that synthetic triterpenoid CDDO derivatives have multiple molecular targets, including signal transducers and activators of transcription (STATs), I $\kappa$ B kinase $\beta$, and mammalian target of rapamycin (mTOR) (Liby et al., 2006; Yore et al., 2006, 2011). In particular, CDDO imidazolide (CDDO$\mathrm{Im}$ ) inhibits the binding of NF- $\kappa \mathrm{B}$ to DNA and subsequent transcriptional activation through the direct interaction with $\mathrm{I} \kappa \mathrm{B}$ kinase $\beta$ to prevent NF- $\kappa \mathrm{B}$ p65 nuclear translocation (Yore et al., 2006). To determine this possibility, we downregulated Nrf2 expression in cultured cardiomyocytes using Nrf2 small interfering RNA (Nrf2 siRNA), and then treated cells with CDDO-Me. The coimmunoprecipitation assay with CBP also demonstrated that CDDO-Me inhibits the binding of $\mathrm{NF}-\kappa \mathrm{B}$ to CBP in the absence of Nrf2 (Supplemental Fig. 3). These previous studies and our current findings suggested that these two potential mechanisms may be involved in the beneficial effects of CDDO-Me on cardiac function in CHF. Although we observed that CDDO-Me increased Nrf2 transcription in sham rat hearts (Fig. 3), Nrf2 transcription was also increased in the $\mathrm{CHF}$ group treated with vehicle alone (Fig. 3A). Although the finding of increased transcription and reduced protein may seem paradoxical, we attribute this to the translational inhibition of Nrf2 mRNA by microRNAs, which is supported by our recent work (Tian et al., 2018) and supported by the expression of downstream targets, such as NQO1 and catalase, which were lower in the vehicle-treated CHF group than in vehicle-treated sham group (Fig. 5). In this regard, CDDO-Me treatment may provide some compensation for the Nrf2 translational inhibition and thus partly restore Nrf2 protein.

The premature termination of CDDO-Me in phase 3 clinical trials was due to side effects for patients with type 2 diabetes mellitus and stage 4 chronic kidney disease. These patients were also observed to show characteristics of heart failure and hospitalization history (Chin et al., 2014b). In the present study, our data clearly demonstrated that low doses of CDDOMe administration still exert therapeutic effects on cardiac function in the $\mathrm{CHF}$ setting, consistent with other preclinical studies (Chin et al., 2014a). 


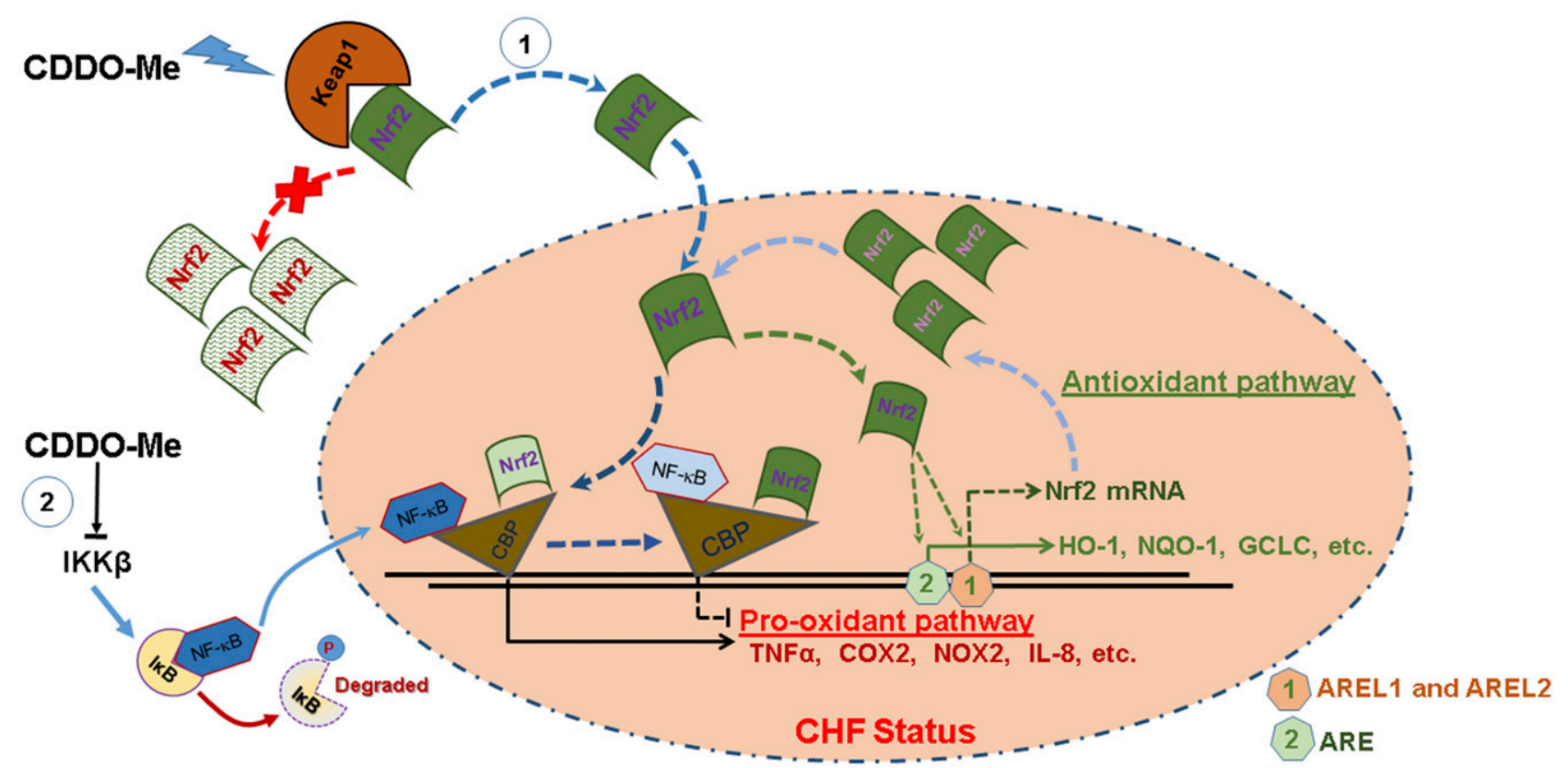

Fig. 8. A schematic diagram of the potential mechanisms for CDDO-Me-mediated Nrf2 activation in CHF. On the one hand, CDDO-Me promotes the dissociation of Nrf2 from Kelch-like ECH-associated protein 1 (Keap1; a natural Nrf2 inhibitor) in the cytosol, thus translocating to the nucleus where enhanced binding of Nrf2 to CBP promotes pro-antioxidant pathways, and reduced binding of NF- $\kappa \mathrm{B}$ to $\mathrm{CBP}$ results in the inhibition of the pro-oxidant pathway in CHF. At the same time, Nrf2 binds to its own promoter at AREL1 and AREL2 sites to amplify its antioxidative effects by increasing its own transcription. On the other hand, CDDO-Me may inhibit the binding of NF- $\kappa \mathrm{B}$ to DNA and subsequent transcriptional activation through the direct interaction with $\mathrm{I} \kappa \mathrm{B}$ kinase (IKK) $\beta$ to prevent NF- $\kappa \mathrm{B}$ p65 nuclear translocation. AREL1 and AREL2, Antioxidant Response Element (ARE)-Like Sequence 1 and 2 .

We must, however, acknowledge some limitations of the current study. First, we only evaluated a relatively short-term effect (2-week administration) of CDDO-Me on cardiac function. The long-term, more-chronic effects of CDDO-Me therapy remain to be investigated. Second, it is unclear why CDDO-Me selectively upregulated the expression of some Nrf2 target genes, including $\mathrm{HO}-1, \mathrm{NQO} 1$, catalase, and glutamatecysteine ligase rather than other putative targets, such as SOD1, SOD2, Grx1, and Gpx1. Third, we initiated CDDO-Me treatment at 12 weeks post MI. It is unclear if the therapeutic effects of CDDO-Me on MI-induced CHF will be determined by different mechanisms when the administration is initiated at different time points.

In conclusion, short-term treatment ( 2 weeks) of CDDO-Me improved cardiac function in CHF rats when administrated at 12 weeks post MI. The significant improvements of oxidative and inflammatory environments in CDDO-Me-treated CHF animals suggest that CDDO-Me can not only modulate the imbalance of free radical generation and elimination by antioxidant defense systems through the upregulation of downstream targets by the enhanced Nrf2 transcription and translation, resulting in the increased binding of Nrf2 to CBP, but also attenuate the production of proinflammatory cytokines through the decreased binding of NF- $\kappa \mathrm{B}$ to CBP. The two potential mechanisms by which CDDO-Me improves heart function are summarized in Fig. 8. This study provides proof of principle that Nrf2 activation may be beneficial in the setting of CHF.

\section{Acknowledgments}

We thank Kaye Talbitzer for her excellent technical assistance in the rodent model of CHF.

\section{Authorship Contributions}

Participated in research design: Tian, Zucker.

Conducted experiments: Tian, Gao, Zhang, Hackfort.

Contributed new reagents or analytic tools: Tian, Gao, Hackfort.

Performed data analysis: Tian, Gao, Hackfort.

Wrote or contributed to the writing of the manuscript: Tian, Zucker.

\section{References}

Appendino G, Belcaro G, Cornelli U, Luzzi R, Togni S, Dugall M, Cesarone MR, Feragalli B, Ippolito E, Errichi BM, et al. (2011) Potential role of curcumin phytosome (Meriva) in controlling the evolution of diabetic microangiopathy. A pilot study. Panminerva Med 53 (3 Suppl 1):43-49.

Ashrafian H, Czibik G, Bellahcene M, Aksentijević D, Smith AC, Mitchell SJ, Dodd MS, Kirwan J, Byrne JJ, Ludwig C, et al. (2012) Fumarate is cardioprotective via activation of the Nrf2 antioxidant pathway. Cell Metab 15: 361-371.

Bai Y, Chen Q, Sun YP, Wang X, Lv L, Zhang LP, Liu JS, Zhao S, and Wang XL (2017) Sulforaphane protection against the development of doxorubicin-induced chronic heart failure is associated with Nrf2 Upregulation. Cardiovasc Ther 35: e12277.

Bai Y, Wang X, Zhao S, Ma C, Cui J, and Zheng Y (2015) Sulforaphane protects against cardiovascular disease via Nrf2 activation. Oxid Med Cell Longev 2015: 407580 .

Bhimaraj A and Tang WH (2012) Role of oxidative stress in disease progression in Stage B, a pre-cursor of heart failure. Heart Fail Clin 8:101-111.

Bubb KJ, Kok C, Tang O, Rasko NB, Birgisdottir AB, Hansen T, Ritchie R, Bhindi R, Reisman SA, Meyer C, et al. (2017) The NRF2 activator DH404 attenuates adverse ventricular remodeling post-myocardial infarction by modifying redox signalling. Free Radic Biol Med 108:585-594.

Camer D, Yu Y, Szabo A, Wang H, Dinh CH, and Huang XF (2016) Bardoxolone methyl prevents the development and progression of cardiac and renal pathophysiologies in mice fed a high-fat diet. Chem Biol Interact 243:10-18.

Canton M, Menazza S, Sheeran FL, Polverino de Laureto P, Di Lisa F, and Pepe S (2011) Oxidation of myofibrillar proteins in human heart failure. $J$ Am Coll Cardiol 57:300-309.

Chin MP, Reisman SA, Bakris GL, O'Grady M, Linde PG, McCullough PA, Packham D, Vaziri ND, Ward KW, Warnock DG, et al. (2014a) Mechanisms contributing to adverse cardiovascular events in patients with type 2 diabetes mellitus and stage 4 chronic kidney disease treated with bardoxolone methyl. Am J Nephrol 39: 499-508.

Chin MP, Wrolstad D, Bakris GL, Chertow GM, de Zeeuw D, Goldsberry A, Linde PG, McCullough PA, McMurray JJ, Wittes J, et al. (2014b) Risk factors for heart failure in patients with type 2 diabetes mellitus and stage 4 chronic kidney disease treated with bardoxolone methyl. J Card Fail 20:953-958. 
Costa S, Reina-Couto M, Albino-Teixeira A, and Sousa T (2016) Statins and oxidative stress in chronic heart failure. Rev Port Cardiol 35:41-57.

Cuadrado A, Rojo AI, Wells G, Hayes JD, Cousin SP, Rumsey WL, Attucks OC, Franklin S, Levonen AL, Kensler TW, et al. (2019) Therapeutic targeting of the NRF2 and KEAP1 partnership in chronic diseases. Nat Rev Drug Discov 18 $295-317$.

de Zeeuw D, Akizawa T, Audhya P, Bakris GL, Chin M, Christ-Schmidt H, Goldsberry A, Houser M, Krauth M, Lambers Heerspink HJ, et al.; BEACON Trial Investigators (2013) Bardoxolone methyl in type 2 diabetes and stage 4 chronic kidney disease. N Engl J Med 369:2492-2503.

Farías JG, Molina VM, Carrasco RA, Zepeda AB, Figueroa E, Letelier P, and Castillo RL (2017) Antioxidant therapeutic strategies for cardiovascular conditions associated with oxidative stress. Nutrients 9:996.

Gao L, Schultz HD, Patel KP, Zucker IH, and Wang W (2005) Augmented input from cardiac sympathetic afferents inhibits baroreflex in rats with heart failure. $H y$ pertension 45:1173-1181.

Gao L, Zimmerman MC, Biswal S, and Zucker IH (2017) Selective Nrf2 gene deletion in the rostral ventrolateral medulla evokes hypertension and sympathoexcitation in mice. Hypertension 69:1198-1206.

Gutierrez J, Ballinger SW, Darley-Usmar VM, and Landar A (2006) Free radicals, mitochondria, and oxidized lipids: the emerging role in signal transduction in vascular cells. Circ Res 99:924-932.

Hu J, Xu Q, McTiernan C, Lai YC, Osei-Hwedieh D, and Gladwin M (2015) Novel targets of drug treatment for pulmonary hypertension. Am J Cardiovasc Drugs 15: $225-234$.

Kim SW, Lee HK, Shin JH, and Lee JK (2013) Up-down regulation of HO-1 and iNOS gene expressions by ethyl pyruvate via recruiting p300 to Nrf2 and depriving it from p65. Free Radic Biol Med 65:468-476.

Kwak MK, Itoh K, Yamamoto M, and Kensler TW (2002) Enhanced expression of the transcription factor Nrf2 by cancer chemopreventive agents: role of antioxidant response element-like sequences in the nrf2 promoter. Mol Cell Biol 22:2883-2892.

Li J, Ichikawa T, Villacorta L, Janicki JS, Brower GL, Yamamoto M, and Cui T (2009) Nrf2 protects against maladaptive cardiac responses to hemodynamic stress. Arterioscler Thromb Vasc Biol 29:1843-1850.

Li W, Khor TO, Xu C, Shen G, Jeong WS, Yu S, and Kong AN (2008) Activation of Nrf2-antioxidant signaling attenuates NFkappaB-inflammatory response and elicits apoptosis. Biochem Pharmacol 76:1485-1489.

Liby K, Voong N, Williams CR, Risingsong R, Royce DB, Honda T, Gribble GW, Sporn MB, and Letterio JJ (2006) The synthetic triterpenoid CDDO-Imidazolide suppresses STAT phosphorylation and induces apoptosis in myeloma and lung cancer cells. Clin Cancer Res 12:4288-4293.

Liu GH, Qu J, and Shen X (2008) NF-kappaB/p65 antagonizes Nrf2-ARE pathway by depriving CBP from Nrf2 and facilitating recruitment of HDAC3 to MafK. Biochim Biophys Acta 1783:713-727.

Lu Z, Xu X, Hu X, Zhu G, Zhang P, van Deel ED, French JP, Fassett JT, Oury TD, Bache RJ, et al. (2008) Extracellular superoxide dismutase deficiency exacerbate pressure overload-induced left ventricular hypertrophy and dysfunction. Hypertension 51:19-25.

Ma A, Hong J, Shanks J, Rudebush T, Yu L, Hackfort BT, Wang H, Zucker IH, and Gao L (2019) Upregulating Nrf2 in the RVLM ameliorates sympathoexcitation in mice with chronic heart failure. Free Radic Biol Med 141:84-92.

Ma Q (2013) Role of nrf2 in oxidative stress and toxicity. Annu Rev Pharmacol Toxicol 53:401-426.

Nagendran J, Norris CM, Appoo JJ, Ross DB, and Nagendran J; Alberta Provincial Project for Outcome Assessment in Coronary Heart Disease (AP PROACH) Investigators (2014) Left ventricular end-diastolic pressure predicts survival in coronary artery bypass graft surgery patients. Ann Thorac Surg 97:1343-1347.

Pergola PE, Raskin P, Toto RD, Meyer CJ, Huff JW, Grossman EB, Krauth M, Ruiz S, Audhya P, Christ-Schmidt H, et al.; BEAM Study Investigators (2011) Bar doxolone methyl and kidney function in CKD with type 2 diabetes. $N$ Engl J Med 365:327-336.

Pickering RJ, Rosado CJ, Sharma A, Buksh S, Tate M, and de Haan JB (2018) Recent novel approaches to limit oxidative stress and inflammation in diabetic complications. Clin Transl Immunology 7:e1016.
Qian Y, Zhong P, Liang D, Xu Z, Skibba M, Zeng C, Li X, Wei T, Wu L, and Liang G (2015) A newly designed curcumin analog Y20 mitigates cardiac injury via antiinflammatory and anti-oxidant actions in obese rats. PLoS One 10:e0120215.

Ruiz S, Pergola PE, Zager RA, and Vaziri ND (2013) Targeting the transcription factor Nrf2 to ameliorate oxidative stress and inflammation in chronic kidney disease. Kidney Int 83:1029-1041.

Santos CX, Anilkumar N, Zhang M, Brewer AC, and Shah AM (2011) Redox signaling in cardiac myocytes. Free Radic Biol Med 50:777-793.

Satta S, Mahmoud AM, Wilkinson FL, Yvonne Alexander M, and White SJ (2017) The role of Nrf2 in cardiovascular function and disease. Oxid Med Cell Longev 2017:9237263.

Shanmugam G, Challa AK, Litovsky SH, Devarajan A, Wang D, Jones DP, DarleyUsmar VM, and Rajasekaran NS (2019) Enhanced Keap1-Nrf2 signaling protects the myocardium from isoproterenol-induced pathological remodeling in mice. Redox Biol DOI: 10.1016/j.redox.2019.101212 [published ahead of print].

Siwik DA, Pagano PJ, and Colucci WS (2001) Oxidative stress regulates collagen synthesis and matrix metalloproteinase activity in cardiac fibroblasts. Am J Physiol Cell Physiol 280:C53-C60.

Siwik DA, Tzortzis JD, Pimental DR, Chang DL, Pagano PJ, Singh K, Sawyer DB, and Colucci WS (1999) Inhibition of copper-zinc superoxide dismutase induces cell growth, hypertrophic phenotype, and apoptosis in neonatal rat cardiac myocytes in vitro. Circ Res 85:147-153.

Tan Y, Ichikawa T, Li J, Si Q, Yang H, Chen X, Goldblatt CS, Meyer CJ, Li X, Cai L, et al. (2011) Diabetic downregulation of Nrf2 activity via ERK contributes to oxidative stress-induced insulin resistance in cardiac cells in vitro and in vivo. $\mathrm{Di}$ abetes 60:625-633.

Tebay LE, Robertson H, Durant ST, Vitale SR, Penning TM, Dinkova-Kostova AT, and Hayes JD (2015) Mechanisms of activation of the transcription factor Nrf2 by redox stressors, nutrient cues, and energy status and the pathways through which it attenuates degenerative disease. Free Radic Biol Med 88 (Pt B):108-146.

Tian C, Gao L, Zimmerman MC, and Zucker IH (2018) Myocardial infarction-induced microRNA-enriched exosomes contribute to cardiac Nrf2 dysregulation in chronic heart failure. Am J Physiol Heart Circ Physiol 314:H928-H939.

Tsutsui H, Ide T, and Kinugawa S (2006) Mitochondrial oxidative stress, DNA damage, and heart failure. Antioxid Redox Signal 8:1737-1744.

Wafi AM, Hong J, Rudebush TL, Yu L, Hackfort B, Wang H, Schultz HD, Zucker IH, and Gao L (2019) Curcumin improves exercise performance of mice with coronary artery ligation-induced HFrEF: Nrf2 and antioxidant mechanisms in skeletal muscle. J Appl Physiol (1985) 126:477-486.

Wang HJ, Pan YX, Wang WZ, Gao L, Zimmerman MC, Zucker IH, and Wang W (2010) Exercise training prevents the exaggerated exercise pressor reflex in rats with chronic heart failure. J Appl Physiol (1985) 108:1365-1375.

Wang YY, Yang YX, Zhe H, He ZX, and Zhou SF (2014) Bardoxolone methyl (CDDO$\mathrm{Me})$ as a therapeutic agent: an update on its pharmacokinetic and pharmacodynamic properties. Drug Des Devel Ther 8:2075-2088.

Xin Y, Bai Y, Jiang X, Zhou S, Wang Y, Wintergerst KA, Cui T, Ji H, Tan Y, and Cai L (2018) Sulforaphane prevents angiotensin II-induced cardiomyopathy by activation of Nrf2 via stimulating the Akt/GSK-3ß/Fyn pathway. Redox Biol 15:405-417.

Yore MM, Kettenbach AN, Sporn MB, Gerber SA, and Liby KT (2011) Proteomic analysis shows synthetic oleanane triterpenoid binds to mTOR. PLoS One 6 e22862.

Yore MM, Liby KT, Honda T, Gribble GW, and Sporn MB (2006) The synthetic triterpenoid 1-[2-cyano-3,12-dioxooleana-1,9(11)-dien-28-oyl]imidazole blocks nuclear factor-kappaB activation through direct inhibition of IkappaB kinase beta. Mol Cancer Ther 5:3232-3239.

Zhang M, An C, Gao Y, Leak RK, Chen J, and Zhang F (2013) Emerging roles of Nrf? and phase II antioxidant enzymes in neuroprotection. Prog Neurobiol 100:30-47.

Zoja C, Benigni A, and Remuzzi G (2014) The Nrf2 pathway in the progression of renal disease. Nephrol Dial Transplant 29 (Suppl 1):i19-i24.

Address correspondence to: Dr. Changhai Tian, Department of Cellular and Integrative Physiology, University of Nebraska Medical Center, 985850 Nebraska Medical Center, Omaha, NE 68198-5850. E-mail: ctian@unmc.edu 